Bond University

Research Repository

\title{
Oral Vitamin C supplements to prevent and treat acute upper respiratory tract infections
}

Van Driel, Mieke L.; Beller, Elaine M.; Thielemans, Emma; Deckx, Laura; Price-Haywood, Eboni; Clark, Justin; De Sutter, An I.M.

Published in:

Cochrane Database of Systematic Reviews

DOI:

10.1002/14651858.CD013292

Licence:

Other

Link to output in Bond University research repository.

Recommended citation(APA):

Van Driel, M. L., Beller, E. M., Thielemans, E., Deckx, L., Price-Haywood, E., Clark, J., \& De Sutter, A. I. M. (2019). Oral Vitamin C supplements to prevent and treat acute upper respiratory tract infections. Cochrane Database of Systematic Reviews, 2019(3), [CD013292]. https://doi.org/10.1002/14651858.CD013292

\footnotetext{
General rights

Copyright and moral rights for the publications made accessible in the public portal are retained by the authors and/or other copyright owners and it is a condition of accessing publications that users recognise and abide by the legal requirements associated with these rights.
}

For more information, or if you believe that this document breaches copyright, please contact the Bond University research repository coordinator. 


\section{(E) Cochrane Library}

Cochrane Database of Systematic Reviews

\section{Oral vitamin C supplements to prevent and treat acute upper respiratory tract infections (Protocol)}

van Driel ML, Beller EM, Thielemans E, Deckx L, Price-Haywood E, Clark J, De Sutter AIM 


\section{TABLE OF CONTENTS}

HEADER . . . . . . . . . . . . . . . . . . . . . . . . . . . . . . . . . . . . . . . 1

ABSTRACT . . . . . . . . . . . . . . . . . . . . . . . . . . . . . . . . . . . . . . 1

BACKGROUND . . . . . . . . . . . . . . . . . . . . . . . . . . . . . . . . . . . . 1

OBJECTIVES . . . . . . . . . . . . . . . . . . . . . . . . . . . . . . . . . . . . . .

METHODS . . . . . . . . . . . . . . . . . . . . . . . . . . . . . . . . . . . . . .

ACKNOWLEDGEMENTS . . . . . . . . . . . . . . . . . . . . . . . . . . . . . . . . . . . . . . .

REFERENCES . . . . . . . . . . . . . . . . . . . . . . . . . . . . . . . . . . . . . . . . . 7

APPENDICES . . . . . . . . . . . . . . . . . . . . . . . . . . . . . . . . . . . . . . . . . . . . . . . . .

CONTRIBUTIONS OF AUTHORS . . . . . . . . . . . . . . . . . . . . . . . . . . . . . . . . . . . . . . . . . . . . . .

DECLARATIONS OF INTEREST . . . . . . . . . . . . . . . . . . . . . . . . . . . . . . . . . . . . 10

SOURCES OF SUPPORT . . . . . . . . . . . . . . . . . . . . . . . . . . . . . . . . . . . . . . . 10 
[Intervention Protocol]

\title{
Oral vitamin C supplements to prevent and treat acute upper respiratory tract infections
}

\author{
Mieke L van Driel ${ }^{1}$, Elaine M Beller ${ }^{2}$, Emma Thielemans $^{3}$, Laura Deckx ${ }^{1}$, Eboni Price-Haywood ${ }^{4}$, Justin Clark ${ }^{2}$, An IM De Sutter ${ }^{5}$ \\ ${ }^{1}$ Primary Care Clinical Unit, Faculty of Medicine, The University of Queensland, Brisbane, Australia. ${ }^{2}$ Centre for Research in Evidence- \\ Based Practice (CREBP), Bond University, Gold Coast, Australia. ${ }^{3}$ Faculty of Science, The University of Queensland, Brisbane, \\ Australia. ${ }^{4}$ The Ochsner Clinical School, The University of Queensland, New Orleans, LA, USA. ${ }^{5}$ Department of Family Medicine \\ and Primary Health Care, Ghent University, Ghent, Belgium
}

Contact address: Mieke L van Driel, Primary Care Clinical Unit, Faculty of Medicine, The University of Queensland, Brisbane, Queensland, 4029, Australia.m.vandriel@uq.edu.au, mieke.vandriel@ugent.be.

Editorial group: Cochrane Acute Respiratory Infections Group.

Publication status and date: New, published in Issue 3, 2019.

Citation: van Driel ML, Beller EM, Thielemans E, Deckx L, Price-Haywood E, Clark J, De Sutter AIM. Oral vitamin C supplements to prevent and treat acute upper respiratory tract infections. Cochrane Database of Systematic Reviews 2019, Issue 3. Art. No.: CD013292. DOI: 10.1002/14651858.CD013292.

Copyright (C) 2019 The Cochrane Collaboration. Published by John Wiley \& Sons, Ltd.

\begin{abstract}
A B S T R ACT
This is a protocol for a Cochrane Review (Intervention). The objectives are as follows:

To assess the effectiveness of oral vitamin $\mathrm{C}$ supplements to prevent and treat acute upper respiratory tract infections in adults and children.
\end{abstract}

\section{B A C K G R O U N D}

\section{Description of the condition}

Upper respiratory tract infections (URTIs) comprise a number of infections involving the nose, sinuses, pharynx, or larynx. Most URTIs are caused by viruses, although bacteria (and much less commonly fungi) can also be involved. Upper respiratory tract infections are common; the 'common cold' (which mainly affects the nasal mucosa) is the most frequent presentation.

The burden of respiratory tract infections on individuals and on society is high. On average, adults experience around 2.5 episodes of each year, and children 3 to 6 episodes (Monto 2002). A UK surveillance report found a weekly incidence of URTIs between 208 and 269 per 100,000 population (RCGP 2011). The total cost of non-influenza viral respiratory illnesses to society is estimated at USD 40,000 million annually, putting costs for respiratory infections above chronic obstructive pulmonary disease or congestive heart failure (Fendrick 2003). Lambert 2008 estimated a mean cost of AUD 309 for all non-influenza URTIs in healthy preschool-aged children.

The common cold is a mild upper respiratory illness that usually affects the nose, sinuses, throat, and larynx (Allan 2014). The predominant symptoms are nasal, such as congestion and rhinorrhoea (nasal discharge), and sneezing, but sore throat and cough are also often present (Allan 2014; Andrewes 1949; Eccles 2005; Heikkinen 2003). A cold usually lasts between 7 and 10 days (Heikkinen 2003). Observational studies estimate that in highincome countries children have around six to eight colds per year, and adults have between two and four annually (Gwaltney 2002; Witek 2015). Common cold is a self limiting and benign illness, 
but due to its high prevalence (especially in autumn and winter), common cold represents an important economic burden through time off work and school, loss of productivity, and money spent on medication and treatment (Bramley 2002; Hellgren 2010). Acute rhinosinusitis is usually preceded by a cold, and viral rhinosinusitis is present in a significant proportion of people with rhinosinusitis-like symptoms (Puhakka 1998). Few people (0.5\% to 2\%) develop bacterial rhinosinusitis (Berg 1986; Gwaltney 1996). Rhinosinusitis is reported to affect approximately $16 \%$ of the adult population in the USA (Anand 2004). Worrall 2011 reported an incidence of acute sinusitis ranging from 15 to 40 episodes per 1000 patients per year, more commonly in adults than in children, whose sinuses are not fully developed. A UK surveillance report found between 0.7 and 1 new cases per 100,000 population per week (RCGP 2011). In a meta-analysis of 29 studies, bacteria were identified in approximately half of all isolates (Shintani Smith 2015).

In acute otitis media, inflammation causes middle ear effusion, resulting in symptoms such as ear pain, otorrhoea, or fever (Lieberthal 2013). The annual incidence of acute otitis media is estimated at $10.85 \%$, representing 709 million cases worldwide, with about $50 \%$ occurring in children under five years of age (Monasta 2012). The highest burden of acute otitis media is found in sub-Saharan Africa, South Asia, and Oceania, where the highest incidence of hearing impairment, a lasting consequence of frequent acute otitis media, is also found (Monasta 2012). Weekly incidence of acute otitis media in the UK ranges from 30 to 40 new cases per 100,000 population (RCGP 2011). There is evidence to suggest that acute otitis media is often a direct result of a respiratory infection (Elliot 2009). Bacteria, viruses, or both bacteria and viruses have been detected in the middle ear fluid of $96 \%$ of people with acute otitis media (e.g. 66\% bacteria and viruses combined, $27 \%$ bacteria alone, and $4 \%$ virus alone) (Lieberthal 2013).

Acute pharyngitis or tonsillitis, or sore throat, is caused by viruses or bacteria or both viruses and bacteria. Weekly incidence of tonsillitis/sore throat in the UK ranges from 55 to 80 per 100,000 population (RCGP 2011). The most common bacterial cause, group A beta-haemolytic streptococcal infection (GABHS or GAS), accounts for $15 \%$ to $30 \%$ of cases in children, and $5 \%$ to $15 \%$ in adults (Vincent 2004). A meta-analysis found a prevalence of GAS in clinical settings of $25.2 \%$ (95\% confidence interval (CI) 23.1 to 27.5 ) in children and $13.7 \%$ (95\% CI 11.1 to 16.8 ) in adults, with a higher prevalence in high-income countries compared with low- to middle-income countries (Oliver 2018). Another metaanalysis of studies in children found a pooled prevalence of GAS of $37 \%$, with $24 \%$ in children aged under five years (Shaikh 2010). Laryngitis presents with a hoarse voice in adults and adolescents and is usually part of a non-specific URTI involving the nose and throat. Laryngitis is mainly caused by virus, although some bacteria can be involved. When the trachea is involved, cough (dry, productive, or both) is the predominant symptom, mostly based on viral infection. In children aged under three years, croup is a common presentation, which involves inflammation of the larynx and trachea following a viral infection. The hallmark symptom is a distinctive barking cough that commonly starts at night and can progress to stridor and respiratory distress (Elliot 2009). Laryngitis in adults is not well reported, but estimates of incidence for all ages from the UK surveillance report range from 6 to 12 per 100,000 population per week (RCGP 2011). Croup typically affects children aged under three years of age and is more common in boys (Denny 1983).

Epiglottitis is an infection that typically affects very young children whose airways are narrow. Epiglottitis can progress to potentially life-threatening airway obstruction. A child with epiglottitis typically presents drooling sitting upright and leaning forward to make breathing easier (Isakson 2011). In countries where children are vaccinated with the Haemophilus influenzae vaccine, the main pathogen involved in epiglottitis, the condition is becoming a rare presentation (Elliot 2009), and is now mostly seen in adults (Isakson 2011). In a 16-year observational study, the overall incidence of acute epiglottitis reduced from 4.5 cases/100,000/year to 0.98 cases $/ 100,000 /$ year after start of the vaccination programme (Isakson 2011).

\section{Description of the intervention}

Vitamin C (ascorbic acid) is a water-soluble vitamin found in fruit and vegetables, particularly in citrus fruit. Vitamin $\mathrm{C}$ plays a crucial role in iron absorption, wound healing, collagen formation, production of neurotransmitters, and in improved glucose metabolism (Yakoot 2011). The recommended dietary intake of vitamin $C$ for adults (aged over 18 years) is $45 \mathrm{mg}$ per day, and for children, $25 \mathrm{mg} /$ day up to 6 months; $30 \mathrm{mg}$ /day between 7 and 12 months; $35 \mathrm{mg} /$ day for 1 to 8 years; and $40 \mathrm{mg} /$ day for children aged 9 to 18 years (Australian Government 2017). Vitamin C deficiency is associated with scurvy. Scurvy is linked with high risk of pneumonia, which in turn has triggered interest in the effect of vitamin $\mathrm{C}$ on respiratory tract infections (Anderson 1974; Hemilä 2009). Vitamin C is acquired through a healthy diet, but a number of dietary supplements are available over the counter. In this review we will consider the efficacy and safety of vitamin $\mathrm{C}$ taken as an oral supplement to a normal diet.

\section{How the intervention might work}

Vitamin C plays a crucial role in iron absorption, wound healing, collagen formation, production of neurotransmitters, and improved glucose metabolism (Yakoot 2011). The mechanism for reduction of the incidence and severity of URTIs may be through an improved immune system. Vitamin $\mathrm{C}$ has antioxidant properties and protects the immune system against oxidative stress generated during infections (Hemilä 2017; Webb 2007). Studies have shown 
that high levels of serum vitamin $\mathrm{C}$ are associated with enhanced antibody response, neutrophil function, and mitogenic response. Furthermore, in vitro models suggest that vitamin $\mathrm{C}$ inhibits rhinoviruses (Webb 2007). The reduction in vitamin C levels observed during infection suggests that supplementing vitamin $\mathrm{C}$ could have a positive impact on preventing and treating infections (Hemilä 2017). This hypothesis is supported by a Swedish cohort study of 2237 adults aged 25 to 64 years showing that women with a high daily intake of vitamin $C$ in their diet ( $\geq 110 \mathrm{mg} /$ day) experienced fewer URTIs over a nine month period than those with lower dietary intake. However, there was no association of vitamin C intake and URTI incidence in men (Raposo 2017). Authors of a systematic review and meta-analysis of vitamin $\mathrm{C}$ supplementation in children concluded that vitamin $\mathrm{C}$ was not effective in preventing URTIs, but reduced URTI duration by approximately 1.6 days (Vorilhon 2018).

In this review, prevention refers to healthy participants with no signs and symptoms of a URTI taking oral vitamin C supplements. Treatment refers to initiating oral vitamin $\mathrm{C}$ supplementation once URTI signs and symptoms arise.

\section{Why it is important to do this review}

Vitamin C achieved popularity as a treatment for the common cold following the 1970 publication of Vitamin Cand the Common Cold (Pauling 1970). Pauling described a meta-analysis of four trials, and concluded there was strong evidence that vitamin $\mathrm{C}$ reduced the incidence and severity of the common cold. Conversely, others have concluded that the apparent benefit of vitamin $\mathrm{C}$ can be explained by the placebo effect (Chalmers 1975; Dykes 1975; Karlowski 1975). Skepticism about the benefits of vitamin C as a treatment for the common cold or other URTIs remains.

A previous Cochrane Review evaluated the effectiveness of vitamin $\mathrm{C}$ for preventing and treating the common cold and found no evidence to support taking vitamin $\mathrm{C}$ to prevent or treat common colds (Hemilä 2013). In light of high consumer interest in overthe-counter treatments such as vitamin $\mathrm{C}$ for a wide range of respiratory infections, this review has a broader perspective and includes URTIs as well as the common cold. We will also include subgroup analyses and report on specific symptoms that may be relevant to patients (e.g. nasal congestion, headache, fever, and sore throat).

\section{O B J E C T I V E S}

To assess the effectiveness of oral vitamin C supplements to prevent and treat acute upper respiratory tract infections in adults and children.

\section{MET HOD S}

\section{Criteria for considering studies for this review}

\section{Types of studies}

We will include double-blind randomised controlled trials (RCTs). We will not consider non-randomised or quasi-randomised trials or unblinded studies because these designs introduce high risk of bias. We will consider cluster-RCTs where participants are randomised at the cluster level and outcomes are measured at the individual level.

We will include studies reported as full text, those published as abstract only, and unpublished data.

\section{Types of participants}

\section{Prevention of URTIs}

We will include healthy adults and children of all ages regardless of gender who have not been diagnosed with a URTI or other respiratory infection.

\section{Treatment of URTIs}

We will include adults and children of all ages regardless of gender with a URTI (symptoms of a URTI for no more than seven days before study commencement). The following URTIs will be eligible for inclusion in the review: common cold, rhinosinusitis, acute otitis media, acute pharyngitis or tonsillitis, laryngitis, tracheitis, or epiglottitis. We will exclude participants with a diagnosis of another upper respiratory condition (such as influenza) or with lower respiratory tract infections.

\section{Types of interventions}

We will include trials comparing oral vitamin $\mathrm{C}$ administration (any dose) daily with placebo, irrespective of usual dietary intake. We will include only placebo-controlled comparisons because studies comparing the intervention with no treatment may overestimate the effect of the active treatment. We will describe the nature of the placebo used because it may be challenging to produce placebos for dissolving tablets. We will consider all time frames for duration of prevention and treatment. 
Types of outcome measures

Primary outcomes

\section{Prevention of URTIs}

1. Incidence of URTIs during the study period (expressed as a proportion of participants who experience a URTI or the number of URTIs over a period of time, or both).

2. Adverse events, such as diarrhoea, nausea, vomiting, heartburn, abdominal symptoms, headache, insomnia, flushing, etc.

\section{Treatment of URTIs}

1. Time to resolution of overall symptoms; we will also consider measures that combine duration and severity (e.g. area under the curve of severity by time).

2. Adverse events, such as diarrhoea, nausea, vomiting, heartburn, abdominal symptoms, headache, insomnia, flushing, etc.

\section{Secondary outcomes}

\section{Prevention of URTIs}

1. Time to occurrence of URTI.

2. Severity of subjective symptoms (overall symptoms, nasal congestion, sneezing, rhinorrhoea, headache, sore throat, and fever). We will also consider measures that combine duration and severity of specific symptoms (e.g. area under the curve of severity by time). Severity may be measured using Likert scales, visual analogue scales (VAS), or other validated instruments.

3. Time to return to work or school.

4. Patient satisfaction.

\section{Treatment of URTIs}

1. Duration of subjective symptoms (nasal congestion, sneezing, rhinorrhoea, headache, sore throat, and fever).

2. Severity of subjective symptoms (overall symptoms, nasal congestion, sneezing, rhinorrhoea, headache, sore throat, and fever). We will also consider measures that combine duration and severity of specific symptoms (e.g. area under the curve of severity by time). Severity may be measured using Likert scales, VAS, or other validated instruments.

3. Time to return to work or school.

4. Patient satisfaction.

Reporting one or more of the listed outcomes in the trial is not an inclusion criterion for the review.

\section{Search methods for identification of studies}

\section{Electronic searches}

We will search the following databases.

- CENTRAL (Cochrane Central Register of Controlled Trials), which contains the Cochrane Acute Respiratory Infections (ARI) Group's Specialised Register

- MEDLINE

- Embase

- CINAHL (Cumulative Index to Nursing and Allied Health Literature)

- Web of Science

We will use the search strategy described in Appendix 1 to search MEDLINE. We will combine the MEDLINE search with the Cochrane Highly Sensitive Search Strategy for randomised trials: sensitivity and precision-maximising version (2008 revision) (Lefebvre 2011). We will modify the MEDLINE search to run in the other listed databases.

We will also conduct searches of the US National Institutes of Health Ongoing Trials Register ClinicalTrials.gov ( clinicaltrials.gov) and the World Health Organization ( WHO) International Clinical Trials Registry Platform ( ICTRP) ( apps.who.int/trialsearch/). We will not impose language or publication restrictions.

\section{Searching other resources}

We will check the reference lists of all primary studies and review articles for additional references. We will contact experts in the field to identify additional unpublished materials.

\section{Data collection and analysis}

\section{Selection of studies}

Three review authors (LD, ET, EB) will independently screen titles and abstracts for inclusion of all the potential studies identified as a result of the search. All citations will be screened by at least two review authors.

We will retrieve the full-text study reports/publications of studies deemed potentially eligible, and three review authors (LD, ET, EB) will independently screen the full texts and identify studies for inclusion, and record reasons for exclusion of the ineligible studies. Any disagreements will be resolved through discussion or by consulting a fourth review author (MVD) if necessary. We will identify and exclude duplicates and collate multiple reports of the same study so that each study, rather than each report, is the unit of interest in the review. We will record the selection process in sufficient detail to complete a PRISMA flow diagram 
and 'Characteristics of excluded studies' table (Moher 2009). We will not impose any language restrictions.

\section{Data extraction and management}

We will use a data collection form for study characteristics and outcome data that has been piloted on at least one study in the review. One review author (ET) will extract study characteristics from the included studies. We will extract the following study characteristics.

1. Methods: study design, total duration of study, details of any 'run in' period, number of study centres and location, study setting, withdrawals, and date of study.

2. Participants: $\mathrm{N}$, mean age, age range, gender, severity of condition, diagnostic criteria, baseline lung function, smoking history, information on usual dietary intake of vitamin C, inclusion criteria, and exclusion criteria.

3. Interventions: intervention, comparison, concomitant medications, and excluded medications.

4. Outcomes: primary and secondary outcomes specified and collected, and time points reported.

5. Notes: funding for trial, and notable conflicts of interest of trial authors.

Three review authors (LD, ET, EPH) will independently extract outcome data from the included studies. We will note in the 'Characteristics of included studies' table if outcome data are not reported in a useable way. Any disagreements will be resolved by consensus or by involving a fourth review author (ADS). One review author (LD) will enter data into Review Manager 5 (Review Manager 2014). We will double-check that data are entered correctly by comparing the data presented in the systematic review with the study reports. A second review author (ET) will spotcheck study characteristics for accuracy against the trial report.

\section{Assessment of risk of bias in included studies}

Three review authors (ET, EB, ADS) will independently assess risk of bias for each study using the criteria outlined in the Cochrane Handbook for Systematic Reviews of Interventions (Higgins 2011). Any disagreements will be resolved by discussion or by involving another review author (MVD). We will assess risk of bias according to the following domains.

1. Random sequence generation.

2. Allocation concealment.

3. Blinding of participants and personnel.

4. Blinding of outcome assessment.

5. Incomplete outcome data.

6. Selective outcome reporting.

7. Other bias.

We will grade each potential source of bias as high, low, or unclear and provide a quote from the study report together with a justification for our judgement in the 'Risk of bias' table. We will summarise the 'Risk of bias' judgements across different studies for each of the domains listed. We will consider blinding separately for different key outcomes, where necessary. Where information on risk of bias relates to unpublished data or correspondence with a trialist, we will note this in the 'Risk of bias' table.

When considering treatment effects, we will take into account the risk of bias for the studies that contribute to that outcome.

\section{Assessment of bias in conducting the systematic review}

We will conduct the review according to this published protocol and report any deviations from it in the 'Differences between protocol and review' section of the systematic review.

\section{Measures of treatment effect}

We will enter the outcome data for each study into the data tables in Review Manager 5 to calculate the treatment effects (Review Manager 2014). We expect that studies will report our primary outcome in different ways.

We will use the following measures of effect for prevention studies.

- Incidence of URTI (primary outcome 1): risk ratio (RR) where the proportion of participants experiencing a URTI is reported, and rate ratio where rates of infections per unit of time are reported.

- Adverse events (primary outcome 2): RR where the proportion of participants experiencing any or a specific adverse event is reported.

- Time to occurrence of URTI (secondary outcome 1): hazard ratios where the data are provided as a time to event.

- Severity of subjective symptoms (secondary outcome 2): standardised mean difference (SMD) where this is reported on a continuous or ordinal scale.

- Time to return to work or school (secondary outcome 3): hazard ratios where the data are provided as a time to event, SMD where this is reported as number of days (or hours).

- Patient satisfaction (secondary outcome 4): RR where the proportion of participants who are satisfied is reported and standardised mean difference (SMD) where patient satisfaction is reported on a continuous scale.

We will use the following measures of effect for treatment studies.

- Time to resolution of URTI (= duration of illness) (primary outcome 1): SMD where this is reported as number of days (or hours).

- Adverse events (primary outcome 2): RR where the proportion of participants experiencing any or a specific adverse event is reported.

- Duration of subjective symptoms (secondary outcome 1): SMD where this is reported as number of days (or hours).

- Severity of subjective symptoms (secondary outcome 2): SMD where this is reported as number of days (or hours). 
- Time to return to work or school (secondary outcome 3): hazard ratios where the data are provided as a time to event.

- Patient satisfaction (secondary outcome 4): RR where the proportion of participants who are satisfied is reported and SMD where patient satisfaction is reported on a continuous scale.

We will undertake meta-analyses only where this is meaningful, that is if the treatments, participants, and the underlying clinical question are similar enough for pooling to make sense.

We will use meta-regression to explore a dose-response relationship if we find a sufficient number of studies. We will illustrate these results in forest plots by subgroup of dose $(\leq 0.45 \mathrm{~g},>0.45 \mathrm{~g})$ (Australian Government 2017).

\section{Unit of analysis issues}

If we find cluster or cross-over trials, we will use the methods recommended in the Cochrane Handbook for Systematic Reviews of Interventions (Higgins 2011). If there are studies with more than one dose used, we will combine all active treatment arms and compare with the placebo/no treatment arm for the main comparisons. However, if possible we may use dose data in analysis of dose-response.

\section{Dealing with missing data}

We will contact investigators or study sponsors to verify key study characteristics and to obtain missing numerical outcome data where possible (e.g. when a study is identified as abstract only). Where this is not possible, and the missing data are thought to introduce serious bias, we will explore the impact of including such studies in the overall assessment of results by a sensitivity analysis. If numerical outcome data such as standard deviations or correlation coefficients are missing and cannot be obtained from the trial authors, we will calculate them from other available statistics, such as $\mathrm{P}$ values, according to the methods described in the Cochrane Handbook for Systematic Reviews of Interventions (Higgins 2011).

\section{Assessment of heterogeneity}

We will use the $\mathrm{I}^{2}$ statistic to measure heterogeneity among the trials in each analysis. If we identify substantial heterogeneity, we will report it and explore possible causes by prespecified subgroup analysis. We will consider an $\mathrm{I}^{2}$ statistic of $50 \%$ or more to represent important heterogeneity. We will explore the causes of statistical heterogeneity using sensitivity analyses. We will not carry out a meta-analysis if heterogeneity is greater than $90 \%$ and there is too much variation in the results, particularly inconsistency in the direction of the effect.

\section{Assessment of reporting biases}

If we are able to pool more than 10 trials, we will create and examine a funnel plot to explore possible small-study and publication biases.

\section{Data synthesis}

We will pool data from studies we judge to be clinically homogeneous using Review Manager 5 software (Review Manager 2014). If more than one study provides useable data for any single comparison, we will perform a meta-analysis. We will present metaanalyses separately for prevention and treatment trials. If metaanalysis is not possible, we will report a summary of data from individual studies. We will use random-effects models, as we expect the ways of measuring the outcome to differ, and there to be a range of doses. We will also use a random-effects model to pool study data reported as time-to-event rates (i.e. for the primary outcome of 'time to resolution of symptoms' in treatment trials and for the secondary outcome 'time to occurrence of URTI' in prevention trials). We will use the log rank method to obtain a hazard ratio as a pooled estimate of effect (Higgins 2011).

\section{GRADE and 'Summary of findings' table}

We will create a 'Summary of findings' table using the following outcomes for the prevention trials:

1. URTI incidence;

2. adverse events (proportion of total adverse events reported);

3. time to occurrence of URTI;

4. patient satisfaction.

For the treatment trials we will include:

1. time to resolution of symptoms;

2. adverse events (proportion of total adverse events reported);

3. duration of symptoms;

4. severity of symptoms;

5. time to return to work or school;

6. patient satisfaction.

We will use the five GRADE considerations (study limitations, consistency of effect, imprecision, indirectness, and publication bias) to assess the quality of a body of evidence as it relates to the studies that contribute data to the meta-analyses for the prespecified outcomes (Atkins 2004). We will use the methods and recommendations described in Section 8.5 and Chapter 12 of the Cochrane Handbook for Systematic Reviews of Interventions (Higgins 2011), employing GRADEpro GDT software (GRADEpro GDT). We will justify all decisions to down- or upgrade the quality of the evidence using footnotes, and we will make comments to aid the reader's understanding of the review where necessary. 


\section{Subgroup analysis and investigation of heterogeneity}

We plan to carry out the following subgroup analyses:

1. adults and children ( $<12$ years of age);

2. specific URTIs (e.g. tonsillitis, otitis media, rhinosinusitis, etc.);

3. different vitamin $\mathrm{C}$ doses (lower or higher than the recommended daily dose, i.e. $\leq 0.45 \mathrm{~g}$ and $>0.45 \mathrm{~g}$ );

4. therapeutic studies with and without concomitant symptomatic medication; and

5. smokers versus non-smokers.

We will use the $\mathrm{Chi}^{2}$ test to test for subgroup interactions in Review Manager 5 (Review Manager 2014).

\section{Sensitivity analysis}

We will perform sensitivity analyses to assess the impact of heterogeneity on the overall outcome (pooled estimate) of the metaanalysis. We will do this by gradually removing single trials to in- vestigate the extent to which they contribute to heterogeneity. We will also use sensitivity analyses to assess the impact of risk of bias on the overall pooled estimate by first pooling the studies with low risk of bias and then gradually adding the studies assessed as having a high risk of bias.

\section{ACKNOWLEDGEMENTS}

The methods section of this protocol is based on a standard template developed by the Cochrane Airways Group and adapted by the Cochrane Acute Respiratory Infections Group.

We gratefully acknowledge feedback from peer reviewers: Associate Professor Gulam Khandaker and Dr Anwar T Merchant (clinical peer reviewers); Ms Ester Martin and Ms Janet Waddell (consumer peer reviewers). We also wish to gratefully acknowledge comments on the draft by Dr Teresa Neeman (Statistical Editor) and Professor Susan Smith (Contact Editor).

\section{R E F E R E N C E S}

\section{Additional references}

\section{Allan 2014}

Allan GM, Arroll B. Prevention and treatment of the common cold: making sense of the evidence. Canadian Medical Association Journal 2014;186(3):190-9.

Anand 2004

Anand VK. Epidemiology and economic impact of rhinosinusitis. Annals of Otology, Rhinology, and Laryngology 2004;113:3-5. DOI: 10.1177/00034894041130S502

Anderson 1974

Anderson TW, Suranyi G, Beaton GH. The effect on winter illness of large doses of vitamin C. Canadian Medical Association Journal 1974;111(1):31-6.

Andrewes 1949

Andrewes $\mathrm{CH}$. The natural history of the common cold. Lancet 1949;1 (6541):71-5.

Atkins 2004

Atkins D, Best D, Briss PA, Eccles M, Falck-Ytter Y, Flottorp S, et al. GRADE Working Group. Grading quality of evidence and strength of recommendations. BMJ 2004; 328(7454): 1490 .

Australian Government 2017

Australian Government. Nutrient reference values for Australia and New Zealand (updated 2017). www.nrv.gov.au/nutrients/vitamin-c (accessed 11 February 2019).

Berg 1986

Berg O, Carenfelt C, Rystedt G, Anggard A. Occurence of asymptomatic sinusitis in common cold and other acute ENT-infections. Rhinology 1986;24(3):223-5.
Bramley 2002

Bramley TJ, Lerner D, Sarnes M. Productivity losses related to the common cold. International Journal of Occupational and Environmental Health 2002;44(9):822-9.

Chalmers 1975

Chalmers TC. Effects of ascorbic acid on the common cold. An evaluation of the evidence. American Journal of Medicine 1975; Vol. 58, issue 4:532-6.

Denny 1983

Denny FW, Murphy TF, Clyde WA Jr, Collier AM, Henderson FW. Croup: an 11-year study in a pediatric practice. Pediatrics 1983;71(6):871-6.

Dykes 1975

Dykes MH, Meier P. Ascorbic acid and the common cold. Evaluation of its efficacy and toxicity. JAMA 1975; Vol. 231, issue 10:1073-9.

\section{Eccles 2005}

Eccles R. Understanding the symptoms of the common cold and influenza. Lancet Infectious Diseases 2005; Vol. 5 , issue 11:718-25.

\section{Elliot 2009}

Elliot AJ, Fleming DM. Common respiratory infections diagnosed in general practice. In: Eccles $\mathrm{R}$, Weber $\mathrm{O}$ editor (s). Common Cold. Basel, Switzerland: Birkhauser Verlag, 2009:47-75.

\section{Fendrick 2003}

Fendrick AM, Monto AS, Nightingale B, Sarnes M. The economic burden of non-influenza-related viral respiratory tract infection in the United States. Archives of Internal Medicine 2003;163:487-94. 
GRADEpro GDT [Computer program]

McMaster University (developed by Evidence Prime). GRADEpro GDT. Version accessed 8 January 2018. Hamilton (ON): McMaster University (developed by Evidence Prime), 2015.

\section{Gwaltney 1996}

Gwaltney JM. Acute community-acquired sinusitis. Clinical Infectious Diseases 1996;1996(23):1209-25.

\section{Gwaltney 2002}

Gwaltney JM. Clinical significance and pathogenesis of viral respiratory infections. American Journal of Medicine 2002; 112(Suppl 6A):13S-8S.

\section{Heikkinen 2003}

Heikkinen T, Järvinen A. The common cold. Lancet 2003; Vol. 361, issue 9351:51-9.

Hellgren 2010

Hellgren J, Cervin A, Nordling S, Bergman A, Cardell LO. Allergic rhinitis and the common cold - high cost to society. Allergy 2010;65(6):776-83.

Hemilä 2009

Hemilä H. Vitamins and minerals. In: Eccles R, Weber

O editor(s). Common Cold. Basel: Birkha“ user, 2009: 275-307.

\section{Hemilä 2013}

Hemilä H, Chalker E. Vitamin C for preventing and treating the common cold. Cochrane Database of Systematic Reviews 2013, Issue 1. DOI: 10.1002/14651858.CD000980.pub4

Hemilä 2017

Hemilä H. Vitamin C and infections. Nutrients 2017;9(4): E339.

Higgins 2011

Higgins JP, Green S, editor(s). Cochrane Handbook for Systematic Reviews of Interventions Version 5.1.0 (updated March 2011). The Cochrane Collaboration, 2011. Available from handbook.cochrane.org.

\section{Isakson 2011}

Isakson M, Hugosson S. Acute epiglottitis: epidemiology and Streptococcus pneumoniae serotype distribution in adults. Journal of Laryngology and Otology 2011;125:390-3.

\section{Karlowski 1975}

Karlowski TR, Chalmers TC, Frenkel LD, Kapikian AZ, Lewis TL, Lynch JM. Ascorbic acid for the common cold. A prophylactic and therapeutic trial. JAMA 1975; Vol. 231, issue 10:1038-42.

\section{Lambert 2008}

Lambert SB, Allen KM, Carter RC, Nolan TM. The cost of community-managed viral respiratory illnesses in a cohort of healthy preschool-aged children. Respiratory Research 2008;9:11. DOI: 10.1186/1465-9921-9-11

\section{Lefebvre 2011}

Lefebvre C, Manheimer E, Glanville J. Chapter 6: Searching for studies. In: Higgins JP, Green S, editor(s). Cochrane Handbook for Systematic Reviews of Interventions Version
5.1.0 (updated March 2011). The Cochrane Collaboration, 2011. Available from handbook.cochrane.org.

\section{Lieberthal 2013}

Lieberthal AS, Carroll AE, Chonmaitree T, Ganiats TG, Hoberman A, Jackson MA, et al. The diagnosis and management of acute otitis media. Pediatrics 2013;131: e964-99.

Moher 2009

Moher D, Liberati A, Tetzlaff J, Altman DG, The PRISMA Group. Preferred reporting items for systematic reviews and meta-analyses: The PRISMA statement. BMJ 2009;339: 2535.

Monasta 2012

Monasta L, Ronfani L, Marchetti F, Montico M, Vecchi Brumatti L, Bavcar A, et al. Burden of disease caused by otitis media: systematic review and global estimates. PLOS ONE 2012;7(4):e36226. DOI: 10.1371/ journal.pone. 0036226

Monto 2002

Monto AS. Epidemiology of viral respiratory infections. American Journal of Medicine 2002;112(6A):4S-12S.

\section{Oliver 2018}

Oliver J, Malliya Wadu E, Pierse N, Moreland NJ, Williamson DA, Bake MG. Group A Streptococcus pharyngitis and pharyngeal carriage: a meta-analysis. PLOS Neglected Tropical Diseases 2018;12(3):e0006335. DOI: 10.1371/journal.pntd.0006335

\section{Pauling 1970}

Pauling L. Vitamin C and the Common Cold. San Francisco (CA): WH Freeman, 1970.

\section{Puhakka 1998}

Puhakka T, Mäkelä MJ, Alanen A, Kallio T, Korsoff L, Arstila P, et al. Sinusitis in the common cold. Journal of Allergy and Clinical Immunology 1998;102(3):403-8.

\section{Raposo 2017}

Raposo SE, Fondell E, Ström P, Bälter O, Bonn SE, Nyrén $\mathrm{O}$, et al. Intake of vitamin $\mathrm{C}$, vitamin $\mathrm{E}$, selenium, zinc and polyunsaturated fatty acids and upper respiratory tract infection - a prospective cohort study. European Journal of Clinical Nutrition 2017;71:450-7. DOI: 10.1038/ ejcn.2016.261

\section{RCGP 2011}

Royal College of General Practitioners. Research \& Surveillance Centre Annual Report 2011. www.rcgp.org.uk/ clinical-and-research/ /media/Files/CIRC/Researchand-Surveillance-Centre/RSC-Annual-Report-2011/ Annual\%20Report\%202011\%20Final\%20(2)/RSC\%20 Annual\%20Report\%202011.ashx (accessed prior to 14 March 2019).

\section{Review Manager 2014 [Computer program]} Nordic Cochrane Centre, The Cochrane Collaboration. Review Manager 5 (RevMan 5). Version 5.3. Copenhagen: Nordic Cochrane Centre, The Cochrane Collaboration, 2014 . 


\section{Shaikh 2010}

Shaikh N, Leonard E, Martin KM. Prevalence of streptococcal pharyngitis and streptococcal carriage in children: a meta-analysis. Pediatrics 2010;126:e557-64. DOI: $10.1542 /$ peds.2009-2648

\section{Shintani Smith 2015}

Shintani Smith S, Henderson Ference E, Evans CT, Tan BK, Kern RC, Chandra RK. The prevalence of bacterial infection in acute rhinosinusitis: a systematic review and meta-analysis. Laryngoscope 2015;125(1):57-69. DOI: 10.1002/lary.24709

\section{Vincent 2004}

Vincent MT, Celestini N, Hussain AN. Pharyngitis. American Family Physician 2004;69(6):1465-70.

\section{Vorilhon 2018}

Vorilhon P, Arpajou B, Vaillant Roussel H, Merlin E, Pereira B, Cabaillot A. Efficacy of vitamin $C$ for the prevention and treatment of upper respiratory tract infection. A meta-analysis in children. European Journal of Clinical
Pharmacology 2018 Nov 21 Epub ahead of print]. DOI: $10.1007 / \mathrm{s} 00228-018-2601-7$

\section{Webb 2007}

Webb AL, Villamor E. Update: effects of antioxidant and non-antioxidant vitamin supplementation on immune function. Nutrition Reviews 2007; Vol. 65, issue 5: 181-217.

\section{Witek 2015}

Witek TJ, Ramsey DL, Carr AN, Riker DK. The natural history of community-acquired common colds symptoms assessed over 4 years. Rhinology 2015;53(1):81-8.

\section{Worrall 2011}

Worrall G. Acute sinusitis. Canadian Family Physician 2011;57(5):565-7.

\section{Yakoot 2011}

Yakoot M, Salem A. Efficacy and safety of a multiherbal formula with vitamin $\mathrm{C}$ and zinc (Immumax) in the management of the common cold. International Journal of General Medicine 2011; Vol. 4:45-51.

* Indicates the major publication for the study

\section{AP PENDICES}

\section{Appendix I. MEDLINE search strategy}

(“Ascorbic Acid”[Mesh] OR “ascorbic acid”[tiab] OR “vitamin c"[tiab] OR “vit c"[tiab])

AND

(“Respiratory Tract Infections”[Mesh] OR Nasopharyngitis[Mesh] OR Rhinitis[Mesh] OR Sinusitis[Mesh] OR Pharyngitis[Mesh] OR Laryngitis[Mesh] OR bronchitis[Mesh] OR bronchiolitis[Mesh] OR "bronchiolitis, viral”[Mesh] OR Pleurisy[Mesh] OR Cough[Mesh] OR Sneezing[Mesh] OR Pneumonia[Mesh] OR "Respiratory Sounds”[Mesh] OR “Otitis Media”[Mesh] OR Earache[Mesh] OR "Influenza, Human" [Mesh] OR "Common Cold”[Mesh] OR "Respiratory infection”[tiab] OR "Respiratory infections" [tiab] OR "Respiratory Tract Infections “[tiab] OR "Respiratory Tract Infection”[tiab] OR “Respiratory inflammation”[tiab] OR "Respiratory tract inflammation” [tiab] OR urti[tiab] OR lrti[tiab] OR ari[tiab] OR nasopharyngitis[tiab] OR rhinopharyngitis[tiab] OR sinusitis[tiab] OR nasosinusitis[tiab] OR rhinosinusitis[tiab] OR rhinitis[tiab] OR rhinorrhoea[tiab] OR rhinorrhea[tiab] OR pharyngitis[tiab] OR "sore throat"[tiab] OR Tonsillitis[tiab] OR laryngitis[tiab] OR croup[tiab] OR pseudocroup[tiab] OR tracheobronchitis[tiab] OR laryngotracheobronchitis[tiab] OR bronchitis[tiab] OR bronchiolitis[tiab] OR pneumonia[tiab] OR pleuropneumonia[tiab] OR bronchopneumonia[tiab] OR pleurisy[tiab] OR cough[tiab] OR Wheeze[tiab] OR Wheezing[tiab] OR “otitis media”[tiab] OR aom[tiab] OR ome[tiab] OR earache[tiab] OR influenza[tiab] OR flu[tiab] OR “common cold”[tiab] OR "common colds" [tiab]) AND

(Randomized controlled trial[pt] OR controlled clinical trial[pt] OR randomized[tiab] OR randomised[tiab] OR placebo[tiab] OR "drug therapy" [sh] OR randomly[tiab] OR trial[tiab] OR groups[tiab])

NOT

(Animals[Mesh] not (Animals[Mesh] and Humans[Mesh])) 


\section{CONTRIBUTIONSOFAUTHORS}

MVD, LD, and EB drafted the protocol.

JC drafted the search strategy.

All authors contributed and approved the final version.

\section{DECLARATIONSOF INTEREST}

Mieke L van Driel: none known.

Elaine M Beller: this review was supported by a Centre for Research Excellence grant from the National Health and Medical Research Council, Australia, to the Centre for Evidence Based Practice, Bond University.

Emma Thielemans: none known.

Laura Deckx: none known.

Eboni Price-Haywood: none known.

Justin Clark: none known.

An IM De Sutter: none known.

\section{SOURCES OF SUPPORT}

\section{Internal sources}

- No financial support received, Other.

\section{External sources}

- Elaine Beller was supported by a Centre for Research Excellence grant from the National Health and Medical Research Council to the Centre for Evidence Based Practice, Bond University, Australia. Salary

- Other authors: No financial support received, Other. 